\title{
A Preclinical Study of Human Embryonic Stem Cell-Derived Mesenchymal Stem Cells for Treating Detrusor Underactivity by Chronic Bladder Ischemia
}

\author{
Hwan Yeul Yu ${ }^{1,2}$ • Jung Hyun Shin ${ }^{1} \cdot$ HongDuck Yun ${ }^{2} \cdot$ Chae-Min Ryu ${ }^{1,2} \cdot$ Seungun Lee ${ }^{2} \cdot$ Jinbeom Heo $^{2} \cdot$ Jisun Lim $^{2}$. \\ Juhyun Park ${ }^{1} \cdot$ Ki-Sung Hong ${ }^{3,4} \cdot$ Hyung-Min Chung ${ }^{3,4} \cdot$ Dong-Myung Shin ${ }^{2}$ (D) $\cdot$ Myung-Soo Choo ${ }^{1}$
}

Accepted: 9 June 2021 / Published online: 29 June 2021

(C) The Author(s) 2021

\begin{abstract}
Background The therapeutic effects of human embryonic stem cell-derived multipotent mesenchymal stem cells (M-MSCs) were evaluated for detrusor underactivity (DUA) in a rat model with atherosclerosis-induced chronic bladder ischemia (CBI) and associated mechanisms.

Methods Sixteen-week-old male Sprague-Dawley rats were divided into five groups $(n=10)$. The DUA groups underwent 30 bilateral repetitions of endothelial injury to the iliac arteries to induce CBI, while the sham control group underwent a sham operation. All rats used in this study received a $1.25 \%$ cholesterol diet for 8 weeks. M-MSCs at a density of 2.5, 5.0, or $10.0 \times 10^{5}$ cells $(250 \mathrm{~K}, 500 \mathrm{~K}$, or $1000 \mathrm{~K} ; \mathrm{K}=$ a thousand) were injected directly into the bladder 7 weeks post-injury, while the sham and DUA group were treated only with vehicle (phosphate buffer solution). One week after M-MSC injection, awake cystometry was performed on the rats. Then, the bladders were harvested, studied in an organ bath, and prepared for histological and gene expression analyses.

Results CBI by iliac artery injury reproduced voiding defects characteristic of DUA with decreased micturition pressure, increased micturition interval, and a larger residual volume. The pathological DUA properties were improved by M-MSC treatment in a dose-dependent manner, with the $1000 \mathrm{~K}$ group producing the best efficacy. Histological analysis revealed that M-MSC therapy reduced CBI-induced injuries including bladder fibrosis, muscular loss, and apoptosis. Transplanted M-MSCs mainly engrafted as vimentin and NG2 positive pericytes rather than myocytes, leading to increased angiogenesis in the CBI bladder. Transcriptomes of the CBI-injured bladders were characterized by the complement system, inflammatory, and ion transportrelated pathways, which were restored by M-MSC therapy.

Conclusions Single injection of M-MSCs directly into the bladder of a CBI-induced DUA rat model improved voiding profiles and repaired the bladder muscle atrophy in a dose-dependent manner.
\end{abstract}

Keywords Detrusor underactivity $\cdot$ Chronic bladder ischemia $\cdot$ Multipotent mesenchymal stem cells $\cdot$ Embryonic stem cells

\section{Introduction}

Detrusor underactivity (DUA) is defined as "low detrusor pressure or short detrusor contraction time, usually in

Hwan Yeul Yu, Jung Hyun Shin and HongDuck Yun contributed equally to this work.

Dong-Myung Shin d0shin03@amc.seoul.kr

Myung-Soo Choo mschoo@amc.seoul.kr

1 Department of Urology, Asan Medical Center, University of Ulsan College of Medicine, 88 Olympic-ro 43-gil, Songpa-gu, Seoul 05505, South Korea combination with a low urine flow rate resulting in prolonged bladder emptying and/or failure to achieve complete bladder emptying within a normal time span" by the international continence society standardization $[1,2]$. To date, the 
available therapeutic options for DUA are suboptimal, with the majority of patients suffering from persistent discomfort, severely deteriorated quality of life, and complications, including recurrent urinary tract infections and urinary retention [3]. Research to develop more effective, long-term therapies for DUA with improved patient outcomes is ongoing.

Stem cell therapy is emerging as a potential treatment option for a wide range of intractable diseases including bladder voiding dysfunction disorders $[4,5]$. Stem cells are characterized as cells that can self-renew and possess differentiation potency, which can be capitalized on to replace the damaged cells. Among the different types of stem cells, mesenchymal stem cells (MSCs) are considered as a reliable source for stem cell therapy. Besides their regenerative capacity, the MSCs can migrate to the damaged tissues and exhibit paracrine effects, such as recruitment of endogenous progenitor cells and secretion of growth-factors that can favor tissue regeneration. MSCs can been isolated from adult or fetal tissues [5], or they can be also derived from pluripotent stem cells (PSCs) such as embryonic stem cells (ESCs) and induced PSCs (iPSCs) [6-8]. The beneficial effects of MSC therapy include the generation of various preclinical bladder dysfunction models and reports of positive patient outcomes from clinical trials [9-17].

Previously, we investigated the therapeutic effects of multipotent MSCs (M-MSCs) derived from human ESCs in a streptozotocin-induced diabetic rat model of DUA. The transplanted M-MSCs integrated into pericytes, which provided favorable micro-environments for detrusor muscle regeneration [12]. In the present study, we aimed to investigate the therapeutic effects of M-MSC treatment in a DUA rat model with extensive vascular endothelial damage of the iliac arteries. Further, we aimed to investigate the underlying pathophysiological mechanisms of DUA for targeted therapeutics.

\section{Methods}

\section{Ethics Statement and Study Approval}

Animal experiments were approved by the Institutional Animal Care and Use Committee of the University of Ulsan College of Medicine (IACUC-2019-12-004) and performed in accordance with the guidelines and regulations.

\section{Study Design}

Sixteen-week-old male Sprague-Dawley rats were divided into five groups: sham $(n=9)$, DUA $(\mathrm{n}=9)$, DUA + 250 K M-MSCs $(n=10)$, DUA $+500 \mathrm{~K} \mathrm{M-MSCs}(n=9)$, and DUA $+1000 \mathrm{~K}$ M-MSCs $(\mathrm{n}=10)(\mathrm{K}=\mathrm{a}$ thousand $)$. All animals were anesthetized by intraperitoneal injection of $30 \mathrm{mg} / \mathrm{kg}$ Zoletil (Virbac Laboratories, Carros, France) prior to operation. Rats in the DUA groups had a 2-French Fogarty arterial embolectomy catheter (E-060-2F; Edwards Lifesciences, Irvine, CA, USA) inserted into the common iliac artery via the femoral artery. The balloon was passed from the common iliac artery to the femoral artery over 30 repetitions in the inflated state, and then the same procedure was repeated bilaterally. The sham group underwent sham operation. All rats received a $1.25 \%$ cholesterol diet (D12336; Research Diets, New Brunswick, NJ, USA) for 8 weeks. Seven weeks after the operation, the DUA plus stem cell treatment groups were injected with $2.5,5.0$, or $10.0 \times 10^{5} \mathrm{M}$-MSCs $(250 \mathrm{~K}$, $500 \mathrm{~K}$, or $1000 \mathrm{~K}$, respectively) directly at the serosa of the anterior bladder wall. For the sham and DUA groups, phosphate-buffer solution (PBS) was injected.

Animals that did not survive in catheter implantation or bladder manipulation were excluded from subsequent analyses. Additional rats $(n=6)$ were used for the organ bath study. The M-MSCs used in the stem cell treatments were maintained up to seven passages only to ensure their functionality as previously described $[9,10,18]$.

\section{Evaluation of Bladder Voiding Function}

One week after M-MSC injection, the experimental groups were evaluated by awake cystometry. Bladder voiding function was evaluated in unrestrained, awake-state rats in metabolic cages as previously described $[9,10,12]$. Detrusor pressure was defined as [intravesical pressure (IVP) - intraabdominal pressure (IAP)]. The contractility of the bladder tissues was measured by the organ bath study, as previously described $[12,19]$. In brief, longitudinal strips of posterior bladder wall were normalized to weight per $1 \mathrm{~g}$ and then mounted in $5 \mathrm{~mL}$ organ baths containing Krebs solution $\left(118 \mathrm{mM} \mathrm{NaCl}, 5.0 \mathrm{mM} \mathrm{KCl}, 2.5 \mathrm{mM} \mathrm{CaCl}_{2}, 1.0 \mathrm{mM}\right.$

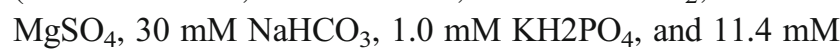
glucose, $\mathrm{pH} 7.4$ ), and maintained at $37{ }^{\circ} \mathrm{C}$ with $5 \% \mathrm{CO}_{2}$ and $95 \% \mathrm{O}_{2}$ continuously supplied. The contractile response to 80 mM KCl (P9333; Sigma-Aldrich, St. Louis, MO, USA), EFS (electrical field stimulation, 1, 2, 4, 8, 16, and $32 \mathrm{~Hz}$ ), 1 mM ATP (A2383; Sigma-Aldrich), or carbachol concentration (PHR1511; Sigma-Aldrich; $1 \mathrm{nM}$ to $1 \mathrm{mM}$ ) were recorded as previously described $[12,19]$. An electrical pulse (1 millisecond pulse width and $80 \mathrm{~V}$ in bath) was delivered using a stimulator (D-7806; Hugo Sachs Elektronik, Germany) for $5 \mathrm{~s}$ at increasing frequencies $(1,2,4,8,16$, and $32 \mathrm{~Hz})$, with 5 min intervals between electrical field stimulations.

\section{Histological and Immunostaining Analysis}

Next, the bladder tissues were harvested for histological and gene expression analyses. For histological analysis, collagen deposition, bladder muscle atrophy, and angiogenesis were assessed with Masson's trichrome staining (Junsei Chemical, Tokyo, Japan) and immunohistochemistry with anti- $\alpha-$ 
smooth muscle actin ( $\alpha$-SMA) (ab7817; Abcam, Cambridge, UK), and anti-CD31 (sc-376,764; Santa Cruz Biotechnology, Dallas, TX, USA) antibody staining, respectively. Apoptosis in each layer of bladder (urothelium and muscle layer) was assessed by terminal deoxynucleotidyl transferase dUTP nickend labeling staining $(1,684,795$; TUNEL, Roche, Mannheim, Germany), and the nuclei were counterstained with 4'6diamnio-2-phenylindole (D9542; Sigma-Aldrich). Two representative areas were selected at random from each slide and used for quantification analysis using Image Pro 5.0 software (Media-Cybernetics, Rockville, MD, USA).

The distribution and cellular properties of transplanted MMSCs were evaluated by immunohistochemical analysis of the bladders with human $\beta 2$-microglobulin (hB2MG) (SC80668; Santa Cruz Biotechnology, Dallas, TX, USA) and by co-staining with antibodies specific for vimentin (\#5741; Cell Signaling Technology, Danvers, MA, USA), $\alpha$-SMA (ab5694; Abcam), NG2 (ab129051; Abcam) and CD31 (ab28364; Abcam). These proteins were visualized by Alexa Fluor 488-conjugated (A11001) or Alexa Fluor 564conjugated (A11010) anti-mouse or anti-rabbit secondary antibodies (Molecular Probes, Grand Island, NY, USA). Images were acquired using a ZEISS LSM800 confocal microscope system (Carl Zeiss, Munich, Germany).

\section{Transcriptome and Gene Expression Analysis}

Publicly available transcriptome datasets (GEO series accession number: GSE122060) [19] were used to compare gene expression data from CBI and sham-operated rat bladders. Differentially expressed genes were determined as 1.5 fold up- or down-regulation, with $p<0.05$ defined as the cutoff values. Transcriptomic features were analyzed using MetaCore (Clarivate Analytics, Philadelphia, PA, USA) with default settings, which provided gene networks, biofunctions, and canonical pathways for representing CBI bladders. Details of the statistical values and significant genes for each analysis are described in the Supplementary Dataset 1. The significance of each candidate gene was individually validated by real-time quantitative PCR (RQ-PCR) analysis, as previously described $[20,21]$. The sequences of primers used in this study were described in the Supplementary Dataset 2.

\section{Statistical Analysis}

Data are reported as the mean standard error of the mean (SEM) and were analyzed using GraphPad Prism 7.0 software (GraphPad Software, La Jolla, CA, USA). Differences and significance were verified by one- or two-way ANOVA followed by Bonferroni post-hoc tests. A $p$ value $<0.05$ was considered as statistically significant.

\section{Results}

\section{Bladder Function Evaluation}

Previously, we reported that DUA could be induced in a rat model of CBI with 30 bilateral repetitions of iliac arterial injury, followed by a $1.25 \%$ high cholesterol diet for 8 weeks [19]. In the present study, we investigated whether M-MSCs derived from human ESCs can show the therapeutic potency for treating DUA in this rat model. To address this issue, we transplanted three different cell dosages $(0.25,0.5$, and $1.0 \times$ $10^{6}$ cells; denoted $250 \mathrm{~K}, 500 \mathrm{~K}$, and $1000 \mathrm{~K}$, respectively) of M-MSCs directly into the bladders at 7 weeks post-CBI injury. As a control, we administered PBS to the CBI-induced DUA rat model (DUA group) and the sham-operated rats (Sham group). In line with our previous report [19], the DUA group presented with decreased detrusor pressure and larger micturition volume, post-void residual, and bladder capacity than the sham group (Fig. 1a and b). A single administration of M-MSCs restored detrusor pressure in a dosedependent manner; however, the beneficial effects of MMSC therapy were suboptimal for restoring micturition interval and volume. Conversely, injection of $1000 \mathrm{~K}$ M-MSCs significantly reduced the residual volume, resulting in decreased bladder capacity, compared with the DUA group (Fig. 1b).

\section{Organ Bath Study}

Bladder strips from the DUA group overall demonstrated significantly deteriorated contractile responses to various stimuli including $80 \mathrm{mM} \mathrm{KCl}$, electrical field stimulation, $1 \mathrm{mM}$ ATP, and carbachol concentration response, compared with the sham group (Fig. 2a-d). In line with the awake cystometry results, M-MSC injection restored these contractility defects in a dose-dependent manner, with $1000 \mathrm{~K} \mathrm{M}$-MSC treatment exhibiting the highest potency in all the stimulations. Taken together, these results demonstrate that a single local administration of $1.0 \times 10^{6} \mathrm{M}$-MSCs was more effective at restoring bladder voiding function and contractibility induced by a high degree of atherosclerotic occlusion.

\section{Histological Analysis}

We next examined whether M-MSC therapy can regenerate the histological damage characteristic of DUA bladders. Rat bladders in the DUA group presented with atrophy of bladder muscle (Fig. 3a) and accompanied fibrosis indicated by accumulation of collagen fibers based on strong Masson's trichrome staining (Fig. 3b). The muscular degeneration was confirmed by immunohistochemical staining of $\alpha$-SMA protein (Fig. 3c). Consistent with these histological injuries, the DUA bladders showed a significant increase of apoptosis in 
Fig. 1 M-MSC injection restored bladder function in DUA rat models. (a)

Representative awake cystometry results and (b) quantitative analysis of bladder voiding parameters 1 week post-injection of either $0.25 \times, 0.5 \times$, or $1.0 \times$ $10^{6} \mathrm{M}-\mathrm{MSCs}(250 \mathrm{~K}, 500 \mathrm{~K}$, or $1000 \mathrm{~K}$ groups, $\mathrm{K}=\mathrm{a}$ thousand) into rat bladders. All quantitative data are presented as mean \pm SEM. * $p<0.05, * * p<0.01$, $* * * p<0.001$ compared with the DUA group; $\# \mathrm{p}<0.05, \# \# \mathrm{p}<$ $0.01, \# \#$ p $<0.001$ compared with the $1000 \mathrm{~K}$ group. One-way ANOVA with Bonferroni posthoc test was used for statistical analysis. IVP, intravesical pressure; IAP, intra-abdominal pressure a
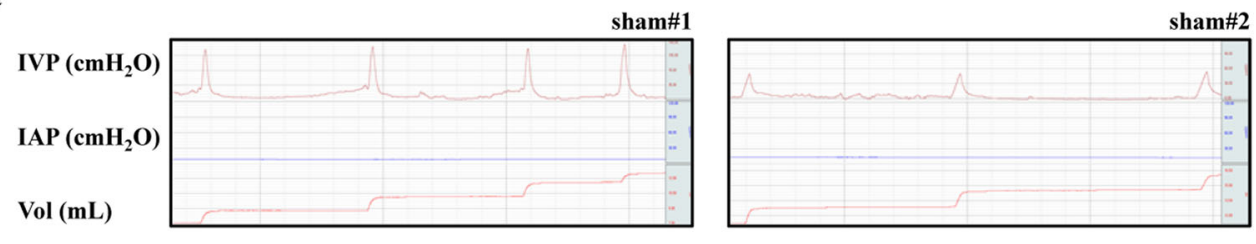

DUA\#1

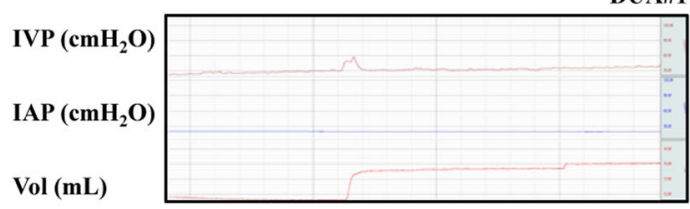

DUA+M-MSC(250K)\#1

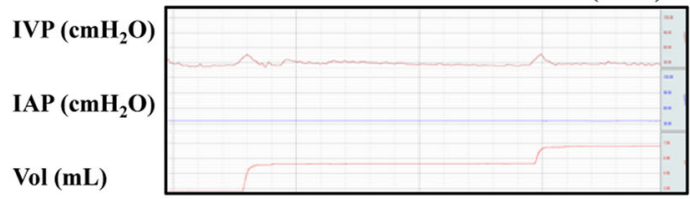

DUA+M-MSC(500K)\#1

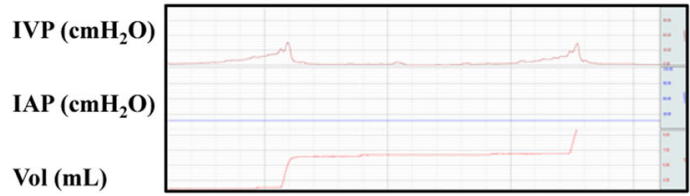

Vol (mL)

DUA+M-MSC(1000K)\#1
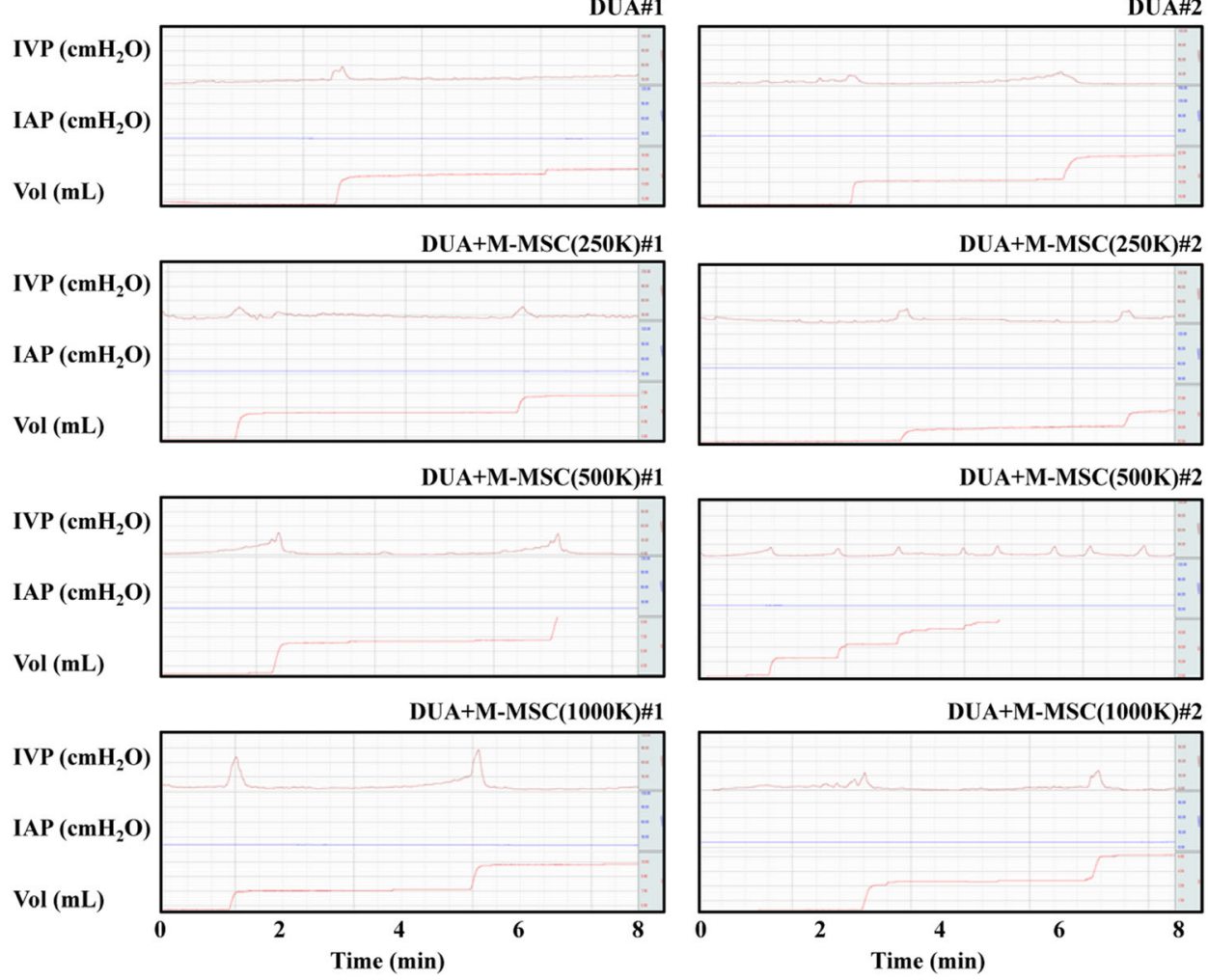

DUA+M-MSC(250K)\#2

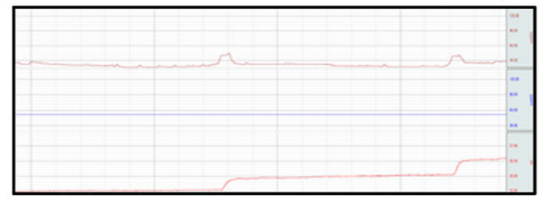

DUA+M-MSC(500K)\#2

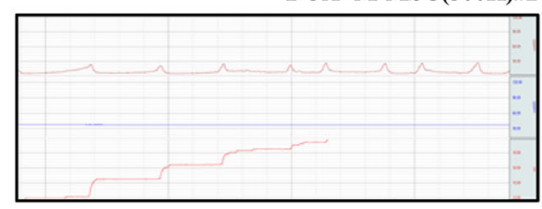

DUA+M-MSC $(1000 K) \# 2$

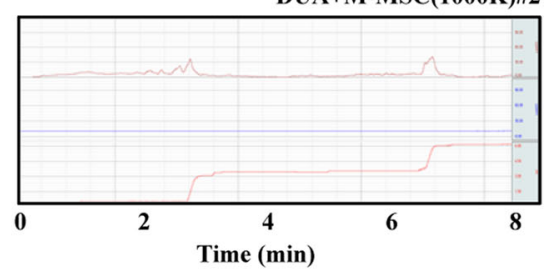

b
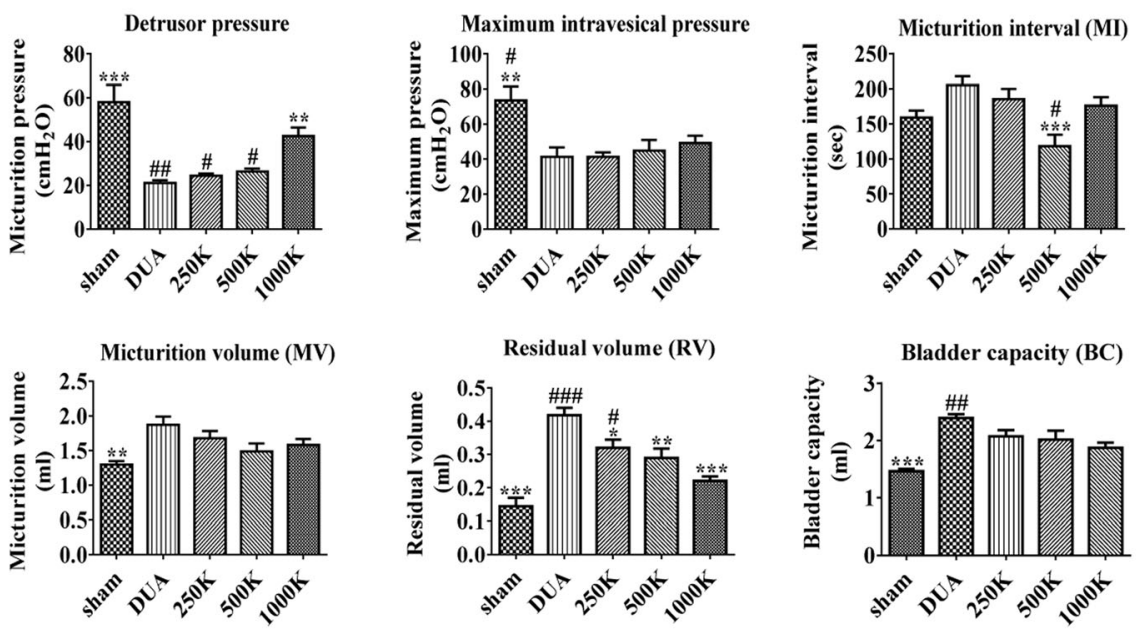

both the urothelial and muscular layer of the bladder along with endothelium of bladder vasculature (Fig. 4a). Of importance, M-MSC injection into the rats alleviated the characteristic DUA histological injuries including fibrosis, muscular degeneration, and increased apoptosis of the bladder muscle tissue (Figs. 3 and 4). Rat bladders treated with
$1000 \mathrm{~K}$ M-MSCs had a significant improvement of tissue fibrosis and muscle atrophy, and reduced apoptosis of muscle fibers, compared with the $250 \mathrm{~K}$ and $500 \mathrm{~K}$ MSC treatment groups (Fig. 3e and d; Fig. 4b-d). These results indicate a dose-dependent therapeutic effect of M-MSC therapy for DUA. 
Fig. 2 Repair of bladder contractility by M-MSC therapy. (a-d) Organ bath analysis for evaluating the contractile response of bladder muscle strips to either $80 \mathrm{mM}$ $\mathrm{KCl}$ (a), electrical field stimulation (b), 1 mM ATP (c), or carbachol, as indicated (d). All quantification data are presented as mean \pm SEM (12 bladder strips from six independent rats). $* \mathrm{p}<$ $0.05, * * \mathrm{p}<0.01, * * * \mathrm{p}<0.001$ compared with the DUA group; $\# \mathrm{p}<0.05, \# \# p<0.01$,

$\# \# \#<<0.001$ compared with the $1000 \mathrm{~K}$ group with one- (a and c) or two- (b and d) ANOVA with Bonferroni post-tests a

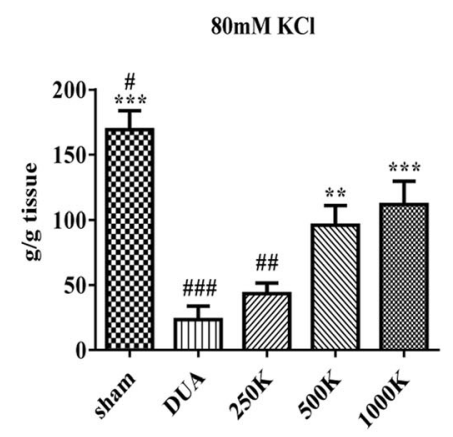

c

$1 \mathrm{mM}$ ATP

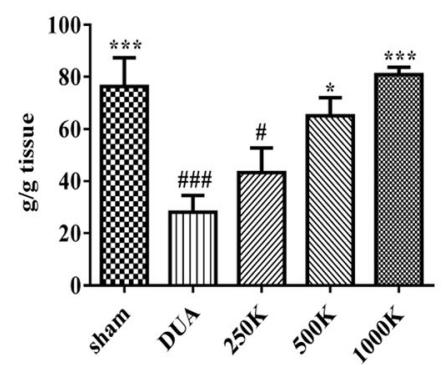

b

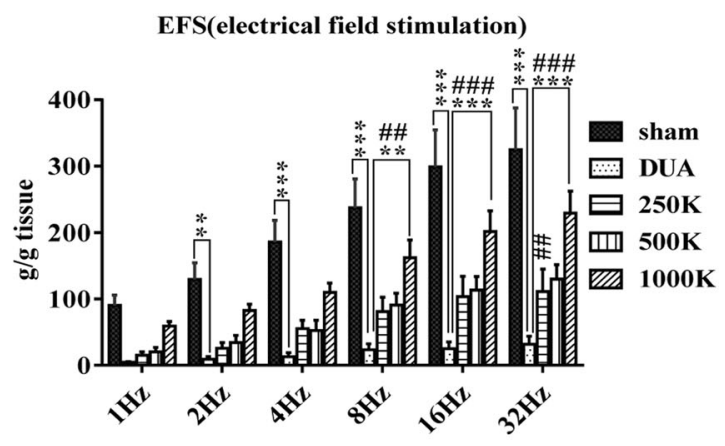

d

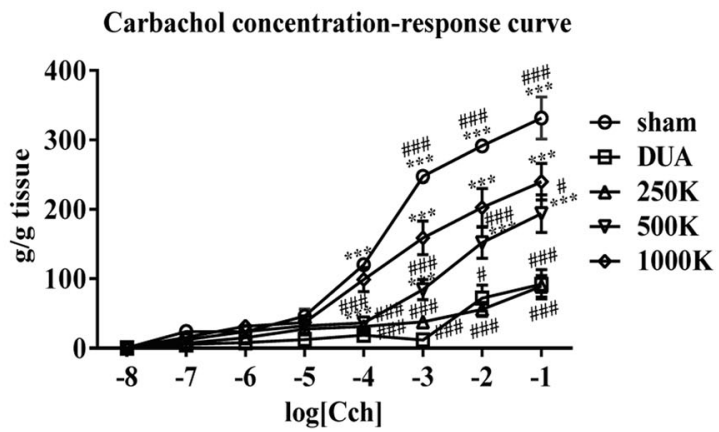

\section{Characterization of Cellular Properties of the Engrafted M-MSCs}

Next, to determine the in vivo distribution and cellular differentiation lineage of the transplanted M-MSCs, the engrafted M-MSCs were detected by immunostaining with the human antigen $\mathrm{hB} 2 \mathrm{MG}$, and co-stained with either $\alpha$-SMA, a muscle marker; vimentin, a mesenchymal marker; or NG2, a pericyte maker. The majority of $\mathrm{hB} 2 \mathrm{MG}^{+}$engrafted cells were localized between muscle and serosa of the bladder at the site of $\mathrm{M}$ MSCs injection. In particular, the engrafted $\mathrm{hB} 2 \mathrm{MG}$ positive $\left({ }^{+}\right)$cells were frequently observed near but not in the muscle fibers, and they expressed minimal $\alpha$-SMA protein (Fig. 5a), suggesting that the transplanted M-MSCs may not contribute toward the myocyte in the CBI-injured bladders. Instead, the $\mathrm{hB}_{2} \mathrm{MG}^{+}$cells were localized in close proximity to bladder vessels and co-stained with the NG2 pericyte and vimentin stromal marker proteins (Fig. 5b and c). The results indicated that M-MSCs mainly engrafted as pericytes to support paracrine effects for repairing tissue damage in the CBI bladders.

\section{The Role of M-MSC Therapy in Angiogenesis of the CBI Bladders}

Induction of angiogenesis is an important mechanism for elucidating the beneficial outcome of MSC therapy [22]. To address this issue, we quantified the blood vessel content in the rat bladders by immunohistochemical analysis with CD31, an endothelial cell marker. In line with the engraftment of MMSCs as a pericyte (Fig. $5 \mathrm{~b}$ and c), the content of CD31 ${ }^{+}$ vessels in the CBI-injured bladders was increased by the administration of M-MSCs (Fig. 6a and b). However, the majority of $\mathrm{hB} 2 \mathrm{MG}^{+}$cells expressed practically no CD31 antigen (Fig. 5d), indicating that pericytes near blood vessels, not endothelial cells, were the major cellular fate of the engrafted M-MSCs in the CBI bladders.

To obtain more mechanistic insights, we examined the expression level of several factors related to angiogenesis and the repair of the bladder injury [4, 23]. The gene expression analysis revealed that M-MSCs, compared with IMR90, a differentially normal fibroblast cell, had significantly increased expression of a subset of pro-angiogenic factors and their cognate receptors, including vascular endothelial growth factor-A (VEGFA), platelet-derived growth factors ( $P D G F-A$, $-B$, and $-D)$, fibroblast growth factor-2 (FGF2), transforming growth factor $\beta-1$ (TGFB1), VEGF receptor-1 (VEGFR1), integrin subunit $\alpha-\mathrm{V}$ (ITGAV), angiopoietin-1 receptor (TEK), and CD44 (Fig. 6c and Suppl Fig. 1). In addition, M-MSCs up-regulated their expression of WNT family member genes (e.g., WNT2, WNT4, and WNT5B), which play an important role in repair of the bladder injury [9, 10, 14, 24]. Taken together, these results support the finding that $\mathrm{M}$ MSCs, mainly engrafted as pericytes in blood vessels near bladder muscle fibers, stimulate angiogenesis in the CBI injured bladder, which might be crucial for their therapeutic potency. 
a
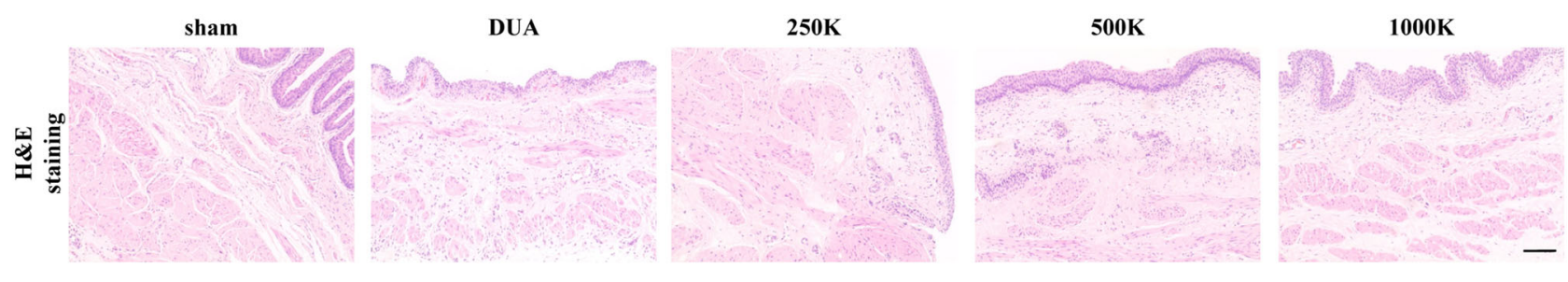

b
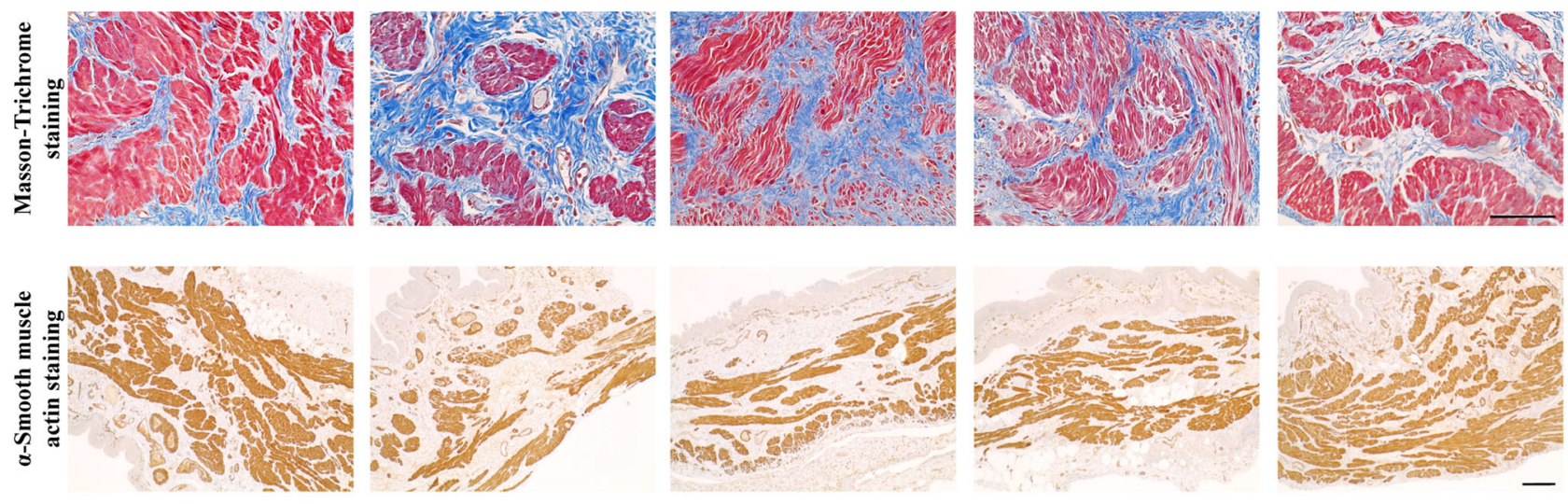

d

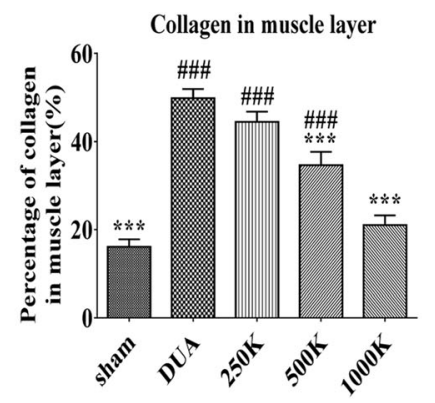

e

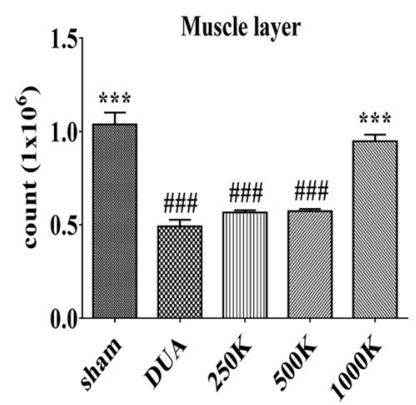

Fig. 3 M-MSCs injection repaired histological injury in CBI bladders. (a and b) Representative images for hematoxylin and eosin (H\&E; magnification $\times 100$, scale bar $=200 \mu \mathrm{m}, \mathbf{A})$ and Masson's trichrome (magnification $\times 400$, scale bar $=200 \mu \mathrm{m}$, b) staining in the bladder tissues 1 week after transplantation of the indicated dosage MMSCs. The tissue fibrosis was stained in blue. (c) Representative images for immuno-histochemical staining of $\alpha$-smooth muscle actin ( $\alpha$-SMA,

\section{Gene Expression Analysis}

A previous transcriptomic study reported that CBI-induced bladder injury characteristically up-regulated the genes involved in the IL-17 and HIF-1 signaling pathways [19]. For example, the expression of NFKB inhibitor-alpha (Nfkbia), C$\mathrm{X}-\mathrm{C}$ motif chemokine ligand-2 ( $\mathrm{Cxcl}-2)$, and S100 calcium binding protein-A9 (S100a9) representing the IL-17 pathway was significantly increased in the CBI-injured bladders. In addition, the expression of interferon gamma receptor-1 (Ifngr-1) and Vegfa were up-regulated as HIF-1 pathways. Of importance, M-MSC therapy significantly prevented the dysfunction of these IL-17- or HIF-1-related genes (Fig. 7a).

To identify the driving genes underlying DUA pathogenesis induced by ischemic vascular injury, we further analyzed magnification $\times 100$, scale bar $=200 \mu \mathrm{m})$ in the indicated bladders. Nuclei were stained with Mayer's hematoxylin. (d and e) Quantification of histological examinations for fibrosis (d) and $\alpha$-SMA stained muscle fiber (e). All quantitative data are presented as the mean $\pm \operatorname{SEM}(n=9$ or 10$) . * p<0.05, * * p<0.01, * * * p<0.001$ compared with the DUA group; \#p $<0.05, \# \# p<0.001, \# \#$ p $<0.001$ compared with the $1000 \mathrm{~K}$ group with one-way ANOVA with Bonferroni post-tests

the published transcriptome datasets for sham and DUA bladders [19] using the MetaCore transcriptome analysis tool that provides the gene networks, biological processes, and pathway maps. Compared with the sham group, CBI bladders exhibited characteristic changes in the complement immune response-, inflammation-, and ion transport-related pathways (Fig. 7b and Suppl Fig. 2a-2c). The molecular features of CBI bladders were elucidated by examining the altered expression of complement factor-B or $-\mathrm{H}(C f b$ and $C f h)$, complement $\mathrm{C} 2$ (C2), Angiopoietin 1 (Angpt-1), colony stimulating factor 3 receptor (Csf3r), C-X-C motif chemokine ligand-3 (Cxcl-3) genes, interleukin-33 (Il-33), and transferrin (Tf) (Fig. 7c. The transplantation of M-MSCs significantly restored the altered expression of $C f h$ and $T f$ as well as the majority of the inflammatory genes in the bladders. 

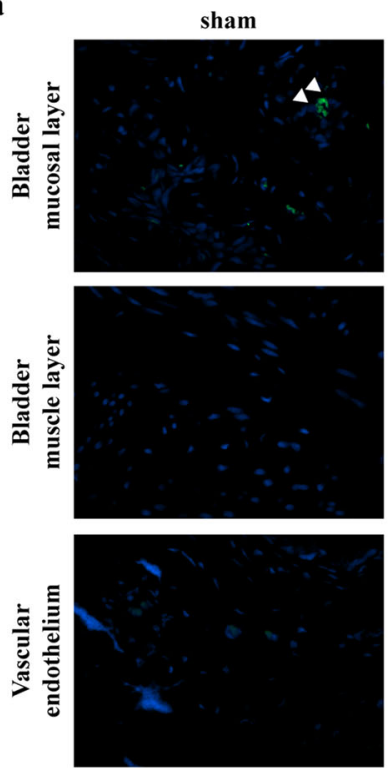

b

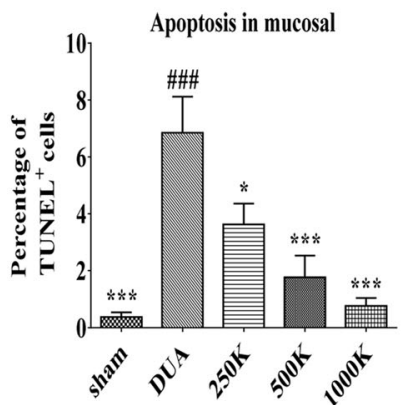

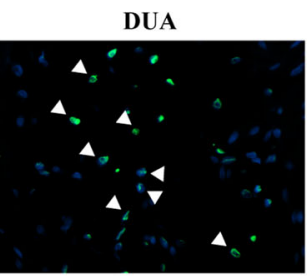
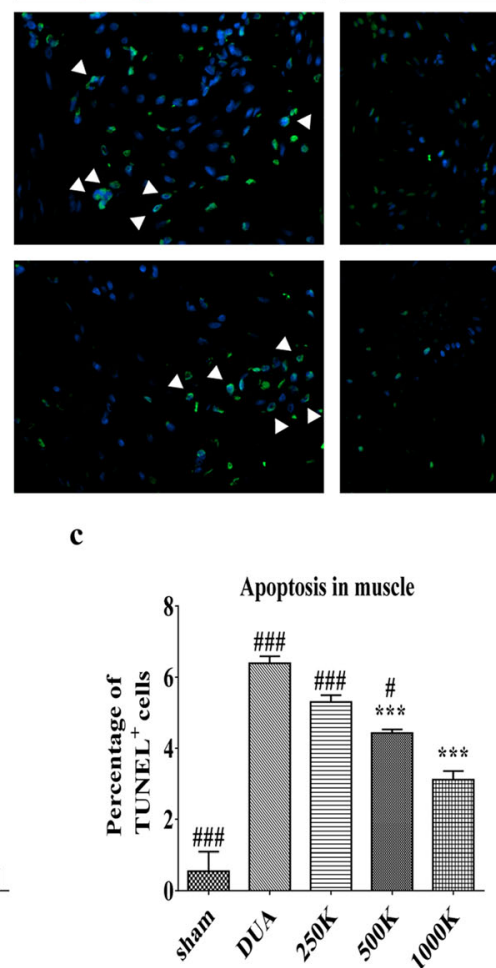
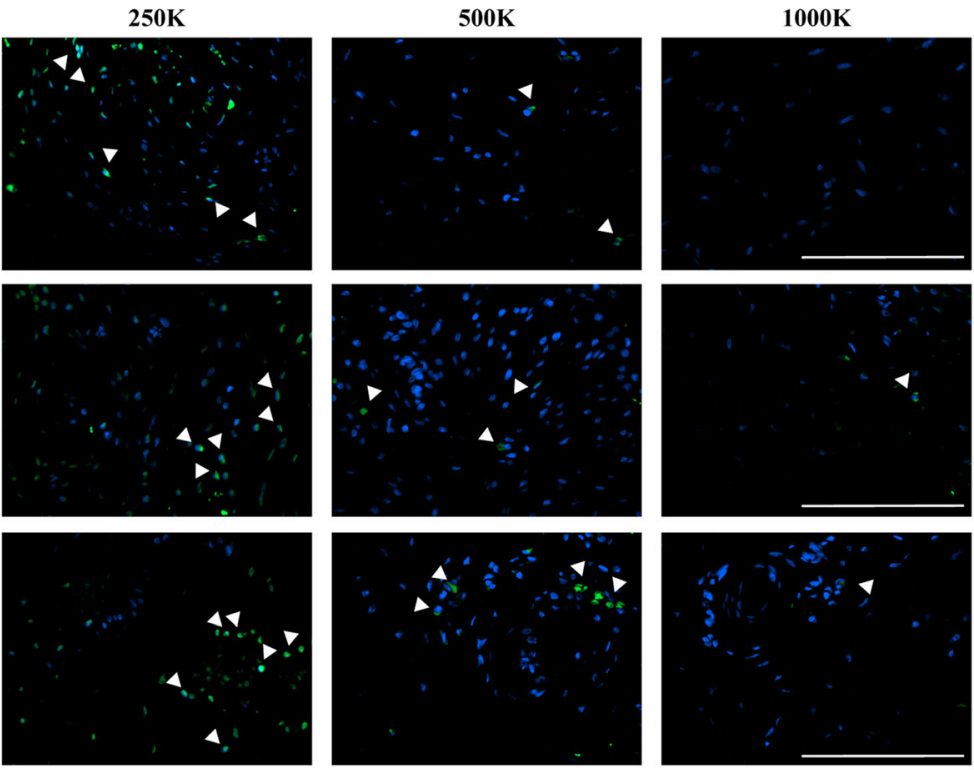

Fig. 4 M-MSC therapy protected the apoptotic response in CBI bladders. (a) Representative images of TUNEL staining for apoptotic cells (green, magnification $\times 400$, scale bar $=200 \mu \mathrm{m}$ ) in the mucosal and muscle layers, and vascular endothelium of bladders in the indicated groups. Nuclei were stained with DAPI. (b-d) Quantification of percentage of apoptotic cells (arrowhead in a) by calculating the ratio

Furthermore, MetaCore gene network analysis revealed that the CBI bladders were characterized by the VEGF-A and $\mathrm{I}-\mathrm{KB}$ associated gene networks involving Vegf-a, Ikb, and the L1 cell adhesion molecule (L1 cam) (Suppl. Fig. 2d), as well as networks related to the sensory perception of chemical stimuli, including claudin-23 (Cldn23), alkylglycerone phosphate synthase (Agps, also known as Adas), Angpt-1, transmembrane Channel Like 7 ( $\operatorname{Tmc} 7$ ), and interferoninduced protein with tetratricopeptide repeats-1 (Ifit-1, also known as Ifi-56) (Fig. 7d). Accordingly, CBI injury significantly down-regulated $C l d n 23$ and up-regulated $T m c 7$ in the bladders treated with M-MSCs (Fig. 7e). Collectively, these results demonstrate the novel significance of the complement system and ion transport-related pathways. Further, these results indicate that CBI-induced DUA pathogenesis leads to an NF-KB- and VEGF-A-mediated inflammatory response, which may be important when considering the applications of M-MSC therapy.

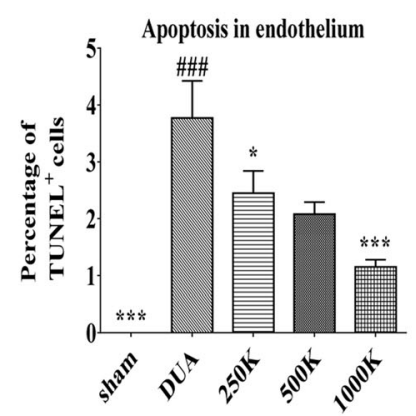

of apoptotic cells (TUNEL positive) to total cells (DAPI-stained nuclei). All quantitative data are presented as the mean $\pm \operatorname{SEM}(\mathrm{n}=9$ or 10$)$. ${ }^{*} p<0.05, * * p<0.01, * * * p<0.001$ compared with the DUA group; \#p $<0.05, \# \# \mathrm{p}<0.001, \# \#$ p $<0.001$ compared with the $1000 \mathrm{~K}$ group with one-way ANOVA with Bonferroni post-tests

\section{Discussion}

In this preclinical study, we demonstrate the therapeutic effects of M-MSC transplantation in a CBI-induced rat model of DUA. Following transplantation, the injected M-MSCs mainly integrated into pericytes nearby muscle fibers of the rat bladders and exhibited paracrine effects to repair vascular insult and detrusor muscle damage. Mechanistically, M-MSC therapy prevented the NF- $\mathrm{kB}$-mediated inflammatory and complement system response in the CBI-induced bladders.

In human DUA patients, a common symptom is an underactive bladder that is characterized by a slow urine stream, hesitancy, and straining to void, with or without a feeling of incomplete bladder emptying [25]. Diagnosis of underactive bladder is frustrating as current therapeutic options have suboptimal and limited efficacy. In the early phase of DUA, patients are able to void with abdominal straining and oral medication but might complain of a weak stream and residual 
Fig. 5 Cellular lineages of transplanted M-MSCs in the CBI bladders. (a-c) Representative confocal microscopic images (magnification $\times 1000$, scale bar $=10 \mu \mathrm{m})$ of human B2MG (hB2MG, green) co-stained with either $\alpha$-SMA myocyte (a), vimentin stromal cell (b), NG2 pericyte (c), or CD31 endothelial cell (d) markers in bladder sections harvested from DUA + $1000 \mathrm{~K}$ rats at 1 week posttransplant. Nuclei were stained with DAPI (blue). Note that MMSCs mainly engrafted as pericytes, not endothelial cells in vessels near muscle fibers in the CBI bladders a
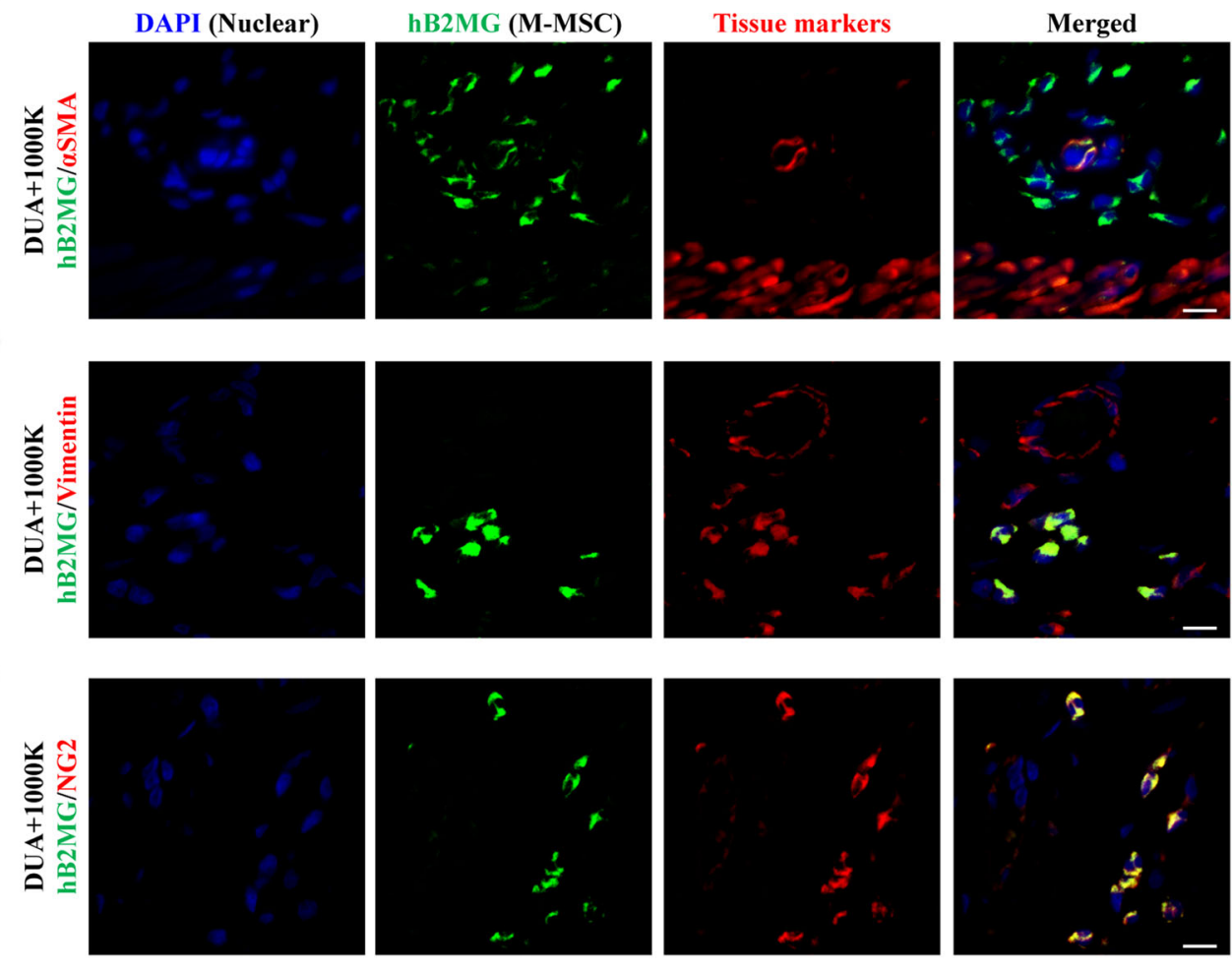

d
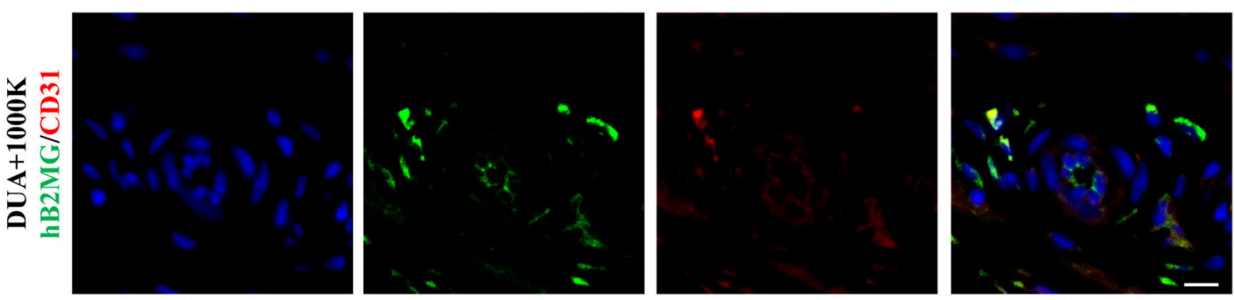

urine sensation. However, as DUA persists, progressive bladder remodeling results in a decompensated state with urothelial dysfunction, neuron and smooth muscle degeneration, and high levels of extracellular matrix deposition in the bladder [26]. Timely intervention to prevent irreversible changes of detrusor is necessary.

The current treatment strategies for DUA aim to improve detrusor contractility, reducing bladder outlet resistance or direct drainage of urine. Surgical intervention to reduce bladder outlet resistance and direct drainage of urine is relatively invasive compared with medical therapy. Direct drainage of urine is not a definite resolution for DUA as it only circumvents the existing problem [27]. In addition, effective bladder emptying cannot be achieved if adequate detrusor contraction is absent. Considering that normal voiding is achieved by adequate, continuous detrusor contraction, which leads to complete bladder emptying within a normal time span, future investigations to fulfill the unmet needs of DUA should focus on restoring the detrusor contractility before the golden time.

Stem cell therapy seems to be an adequate candidate for restoring detrusor contractility [4, 12]. Contemporary concepts in the pathogenesis of DUA are complex, such that its etiological factors can be classified as idiopathic, neurogenic, myogenic, iatrogenic, and functional [28]. Our CBI DUA model presented with myogenic degeneration, increased apoptosis in both the urothelial and muscular layers of the bladder, and increased collagen deposition. A single administration of M-MSCs successfully alleviated both the histological and functional abnormalities by reversing muscle atrophy and reducing the inflammatory and complement system response in CBI-induced rat bladders. Therefore, the present study provides an in vivo proof of concept that MSC therapy is a viable option for treating DUA.

The application of autologous muscle-derived mesenchymal stem cells (AMDC) has been reported in several clinical trials to treat stress urinary incontinence patients. Recently, Gilleran et al., reported the first regulatory approved clinical trial, which evaluated the safety and efficacy of intradetrusor injected AMDCs in 20 non-neurogenic DUA patients [29]. The study subjects received approximately 30 transurethral injections of $0.5 \mathrm{~mL}$ delivered to the bladder (125 million AMDC/15 mL). The initial end point was post-injection 6- 

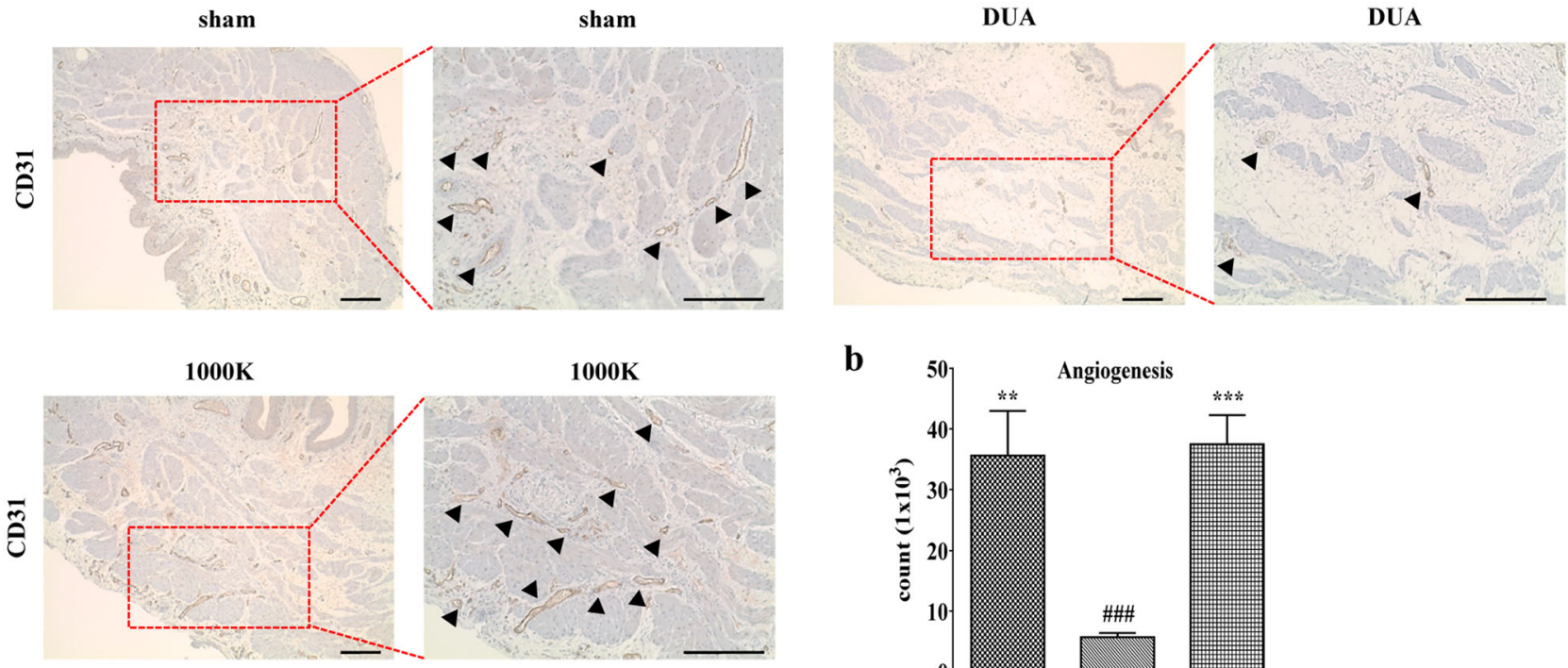

b

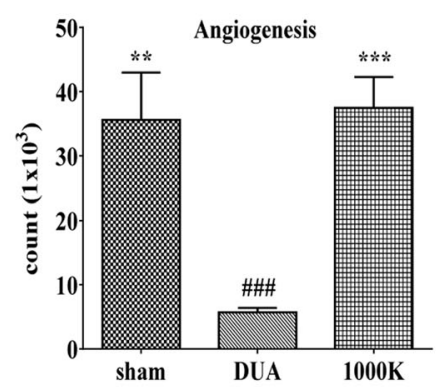

c
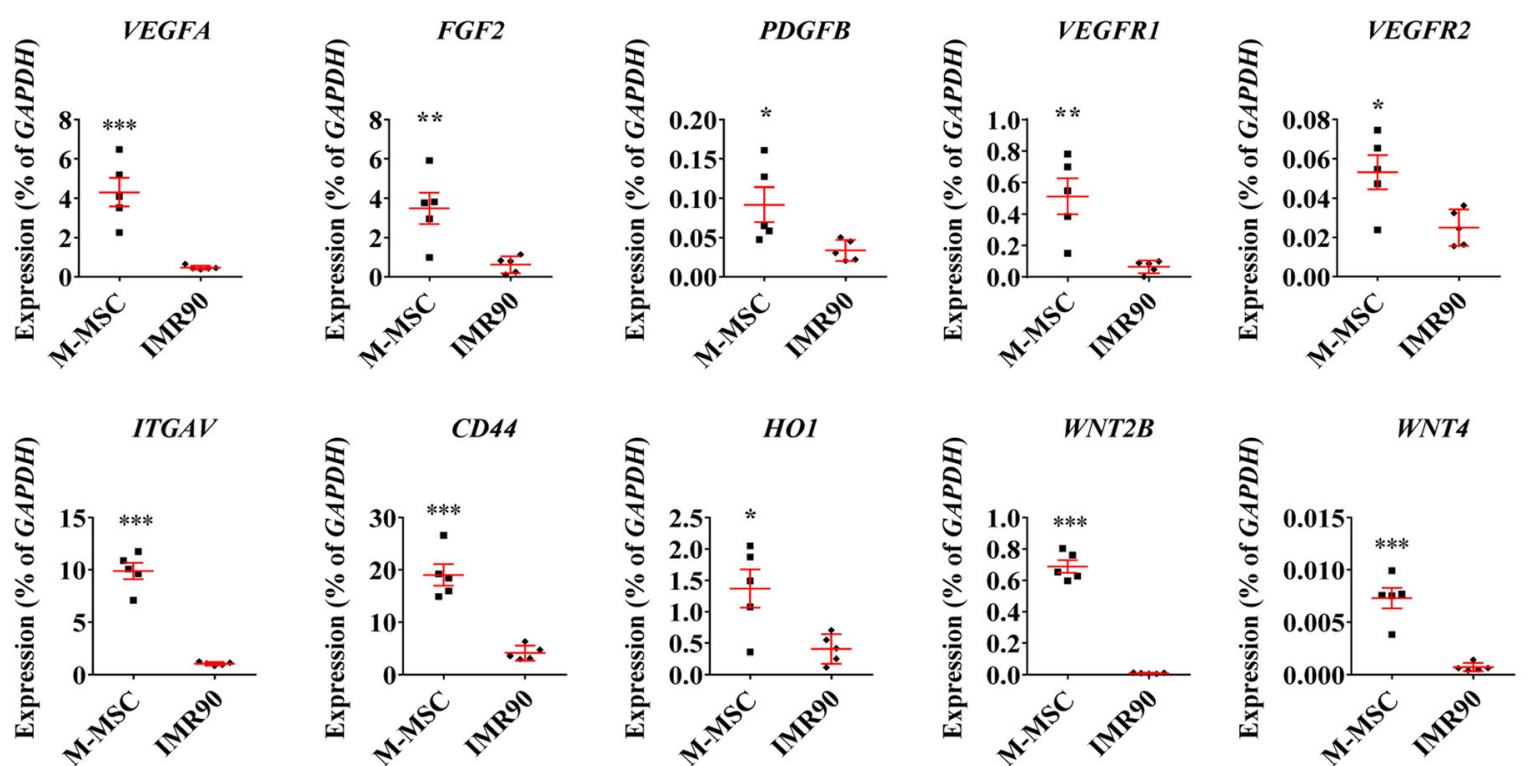

Fig. 6 M-MSCs injection stimulated angiogenesis in CBI bladders. (a) Representative images of immuno-histochemical staining of CD31 (magnification $\times 100$, scale $b a r=200 \mu \mathrm{m}$ ) in the bladder tissues 1 week after transplantation of the indicated dosage of M-MSCs. Photomicrographs with higher magnification (magnification $\times 200$, scale bar $=200 \mu \mathrm{m}$ ) are shown in the right corner of each panel. The arrowheads indicate the $\mathrm{CD} 31$ positive $\left(\mathrm{CD} 31^{+}\right)$vessels. The nuclei were stained with Mayer's hematoxylin. (b) Quantification of the $\mathrm{CD} 31^{+}$vessels in the indicated bladders. Quantitative data are presented

as the mean \pm SEM ( $\mathrm{n}=9$ or 10$)$. $* * \mathrm{p}<0.01, * * * \mathrm{p}<0.001$ compared with the DUA group; \#\#\#p $<0.001$ compared with the $1000 \mathrm{~K}$ group with one-way ANOVA with Bonferroni post-tests. (c) Expression of transcripts of genes related to the angiogenesis and WNT signaling pathways in the M-MSCs and IMR90, a human primary lung fibroblast. Expression is presented as $\% G A P D H$ and shown as a dot plot of mean \pm SEM $(n=5)$. * p $<0.05, * * \mathrm{p}<0.01, * * * \mathrm{p}<0.001$ compared by the non-parametric Mann-Whitney $U$ test

months; however, all participants asked for a second injection due to satisfactory results so the follow-up assessments were made at post-injection 1, 3, 6, and 12 months. At the primary outcome point of 12 months, 11 out of 19 patients (58\%) reported a global response assessment $\geq 5$, showing slight to marked improvement in their symptoms. In addition, improvement of voiding efficiency was observed in many subjects who were catheter dependent at baseline. No AMDC-related serious adverse events were reported. The reported adverse events included injection related and biopsy related complications. The main differences between this clinical trial and our preclinical study are the injection route and 

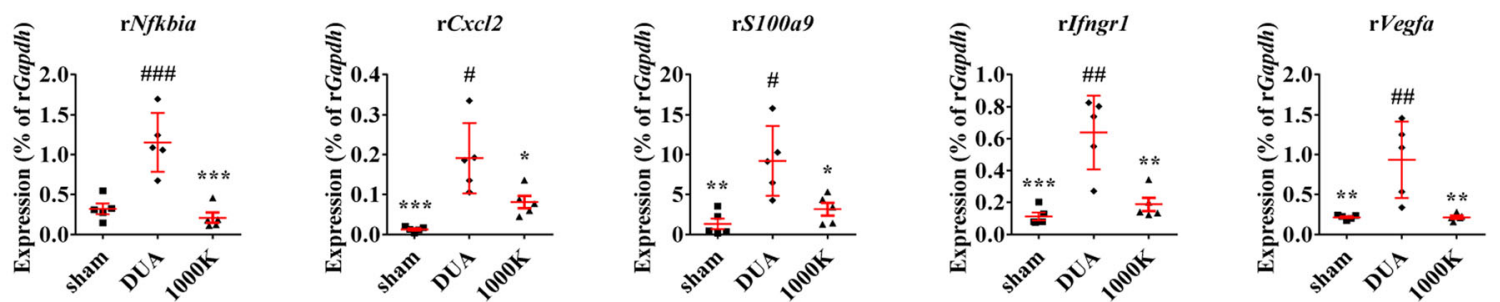

b

Enrichment by Pathway Maps

Alternative complement cascade disruption in age-related macular degeneration Immune response_Alternative complement pathway Development_Cross-talk between VEGF and Angiopoietin 1 signaling pathways Resolution of inflammation in healing myocardial infarction Role of adipose tissue hypoxia in obesity and type 2 diabetes IL-1 signaling in melanoma

Muscle contraction Relaxin signaling pathway Immune response Lectin induced complement pathway Immune response_Lysophosphatidic acid signaling via NF-kB Immune response_Classical complement pathway

c
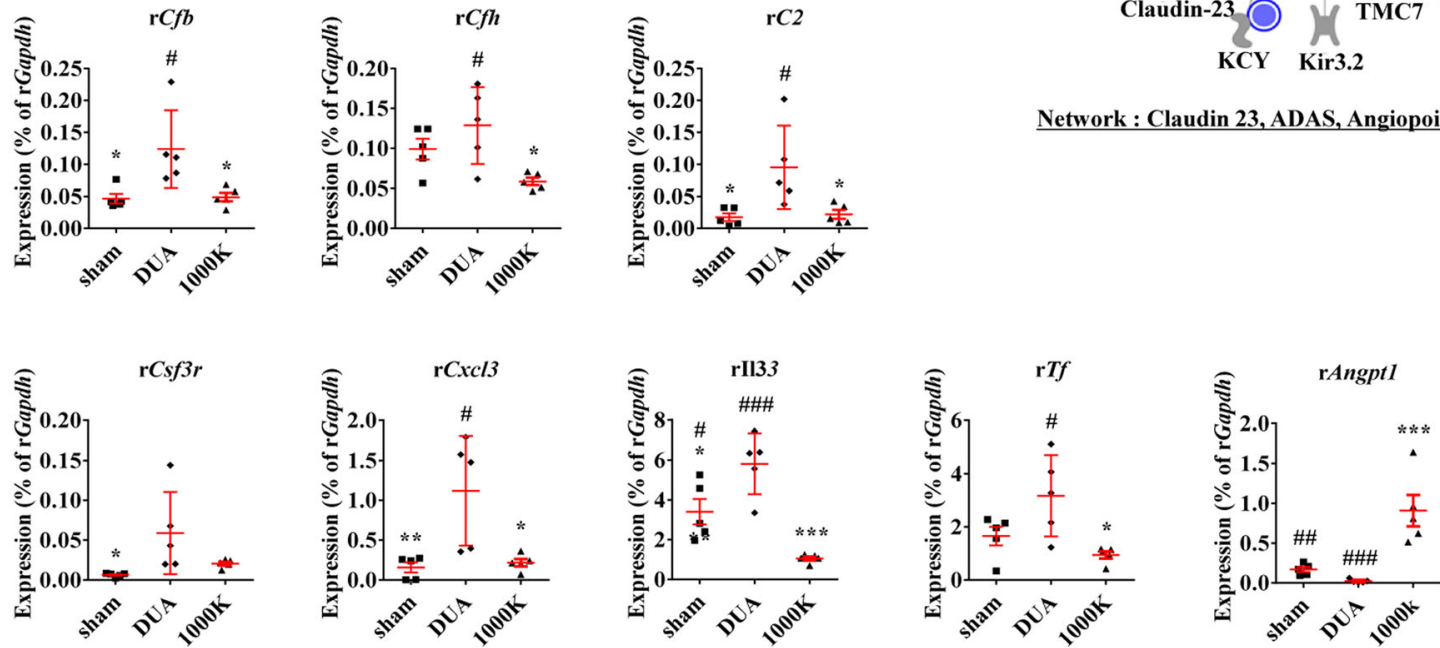

$\mathbf{e}$
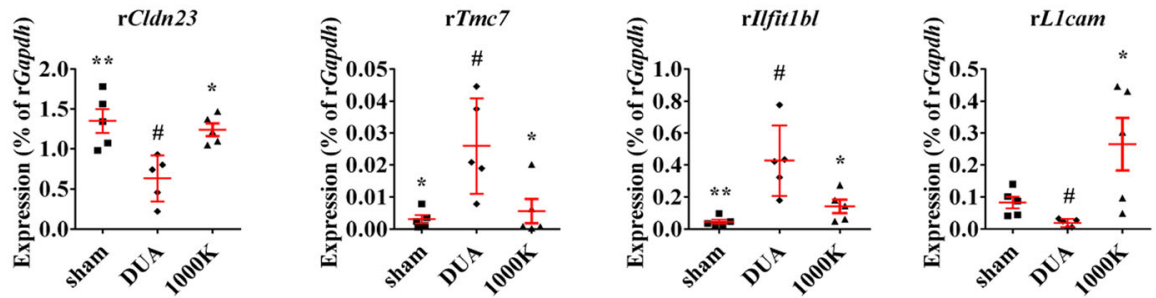

Fig. 7 Gene expression change in CBI-induced DUA bladders following M-MSC therapy. (a) Real-time qPCR analysis of genes relating to the IL-17 and HIF-1 signaling pathways in the indicated bladders. (b and d) MetaCore analysis of DUA and sham bladders revealed the top ten pathway maps (b) and a representative gene network associated with the sensory perception of chemical stimuli (d). The gene network is illustrated by overlaying experimental values as fold changes in CBI versus sham samples. Up- and down-regulated genes are d

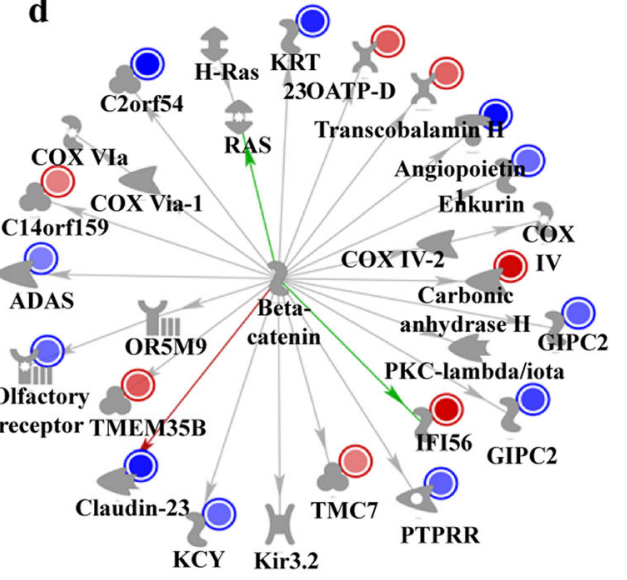

Network : Claudin 23, ADAS, Angiopoietin 1, TMC7, IFI56 indicated in red and green, respectively. (c and e) Real-time qPCR analysis of genes related to the complement system, inflammatory, and ion transport-related pathways (d), as well as genes involved in networks characteristic of CBI (e) in the indicated bladders. Expression is presented as \% of Gapdh expression and shown as a dot plot of mean SEM $(n=5)$. $* \mathrm{p}<0.05, * * \mathrm{p}<0.01, * * * \mathrm{p}<0.001$ compared with the DUA group; \#p $<$ $0.05, \# \# p<0.01, \# \# \# p<0.001$ compared with the $1000 \mathrm{~K}$ group with one-way ANOVA with Bonferroni post-tests 
the type of stem cells used. As there are no currently available devices, such as a cystoscope with an injection channel for rats, the M-MSCs were directly injected into the rat bladder. However, if our study is clinically applied, M-MSCs will be injected via a transurethral route as it is familiar to urologists and is minimally invasive.

Large-scale production of MSCs from adult tissues adversely resulted in the loss of their primitive functions. In addition, adult-tissue derived MSCs, especially those from aged donors, have limited proliferative capacity in vitro due to replicative senescence [30-33], and MSCs at high passage number are more likely to trigger an innate immune attack upon transplantation [34]. To overcome these issues, MMSCs used in this study were derived from hESCs, which have been suggested as a cost-effective alternative source due to their pluripotency and unlimited expansion potential [6]. Indeed, the M-MSCs used in this study could be expanded for more than 30 passages without adverse genetic or functional abnormalities and exhibited several typical MSC features, including fibroblast-like morphology and expression of surface markers characteristic to MSCs (CD73 and CD105) or pericytes (PDGFRB, CD146, and NG2), and chondrogenic, osteogenic, and adipogenic differentiation ability [9]. More importantly, M-MSCs showed superior therapeutic efficacy and long-term in vivo engraftment to adult-tissue counterparts in several animal models of interstitial cystitis/bladder pain syndrome (IC/BPS) [9, 10, 13], ketamine cystitis [11], and diabetes mellitus associated DUA, as well as asthma [33, 35]. Moreover, hESC-derived M-MSCs do not need tissue biopsy or sampling from subjects in a clinical setting, so biopsy-related adverse events can be avoided. In the present study, we demonstrate that hESC-derived M-MSCs can be also effective for treating DUA induced by chronic vascular endothelial damage.

In several preclinical and clinical trials using adult-tissue derived MSCs, poor engraftment and survival of the transplanted cells under in vivo conditions have impeded transferring MSC therapy into clinical practice. The preceding data reporting advantages of M-MSCs might be attributable to enhanced in vivo engraftment and survival $[9,10]$. In the CBI-injured bladders, $\mathrm{hB} 2 \mathrm{MG}^{+}$engrafted cells were detected 7 days after transplantation, mainly located between the muscle and serosa of the bladder. The engrafted M-MSCs contributed little to the $\alpha$-SMA ${ }^{+}$myocyte. Instead, they were engrafted into pericytes or stromal cells near blood vessels and muscle fibers, suggesting that their prolonged paracrine effects repair the damaged muscle fibers. The several trophic factors secreted by MSCs are responsible for paracrine effects by mediating immune-suppressive, anti-inflammatory, and pro-angiogenic responses, which have significantly contributed to the beneficial outcomes of MSC therapy targeting several diseases. Gene expression analysis revealed that MMSCs, compared with the differentiated fibroblast cell line, up-regulated the expression of several angiogenic genes (e.g., $V E G F A, P D G F-A$, and $F G F 2$ ) and tissue regenerating WNT family genes (e.g., WNT2B and WNT4). WNT signaling plays a key role in angiogenesis and vascular remodeling or maturation in the tissue development, homeostasis and repair processes [36-39]. In particular, the secreted Frizzled-related protein-1, a modulator of the WNT pathway, stimulates the angiogenic functions of MSCs, leading to vessel maturation and functionality. Therefore, further in-depth characterization of the paracrine factors of M-MSCs, including WNT related factors, could advance our understanding of the mode of action of M-MSC therapy targeted to DUA. In addition, further study is warranted to investigate the underlying mechanisms and key player(s) that modulate the perivascular engraftment of M-MSCs in the DUA pathological condition.

To date, the pathophysiology of CBI-induced DUA has not been fully elucidated $[40,41]$. In the present study, transcriptome analysis of CBI-injured bladders was characterized by the alternation of the complement system (e.g., $C f b, C f h$, and $C 2$ ), and the inflammatory (e.g., Csf $3 r, C x c l-3$, and Il-33), ion transport (Tf), IL-17 (Nfkbia, Cxcl2, and S100a9), and HIF-1 (Vegfa and Angpt-1) signaling pathways. In response to CBI damage, VEGF and ANGPT-1 signaling can cross-talk to stimulate NF-KB activation, enhancing leukocyteendothelial adhesion and aggravating vascular endothelial damage (Suppl. Fig. 3). Furthermore, inflammatory signals, including interleukin-1, can activate VEGF-A expression and angiogenesis in the tumor micro-environments [42-44]. Since M-MSC therapy effectively restored the dysfunction of genes related to VEGF-A and NF- $\mathrm{KB}$ signaling, a further study is required to determine whether the interplay between these signaling pathways is involved in CBI-induced DUA. It would also be interesting to further investigate the role of the complement system in the pathophysiology of DUA, which is relatively unknown.

The main limitation of current study is its preclinical design. The pathophysiology of DUA is multifactorial and our CBI model might not reflect the entire and complex pathogenesis. In addition, despite the promising results from this preclinical study, one major obstacle to the therapeutic application of hESC-derivatives is the safety issues, including the possible formation of teratoma or other tumors, immune rejection, and the risk that the cell will differentiate into unwanted cell types [45]. However, recent successful clinical studies for the therapy of eye disorders could alleviate this general concern of hESC-based therapeutics [46, 47]. Likewise, the aforementioned adverse outcomes were not detected in longterm monitoring up to 1 year in both acute and chronic IC/BPS animal models treated with M-MSC therapy $[9,10]$. However, safety issues surrounding hESC-based therapies must still be thoroughly investigated before clinical application of these cells [45]. 
In conclusion, our findings provide an in vivo proof of concept for treating CBI-induced DUA with hESC-derived M-MSCs that restore bladder voiding functions, contractibility, and histological features. Further, we optimized dosage and elucidated the underlying molecular mechanisms of MMSC therapy.

Supplementary Information The online version contains supplementary material available at https://doi.org/10.1007/s12015-021-10204-z.

Acknowledgments This research was supported by a grant from the Korean Health Technology R\&D Project, Ministry of Health \& Welfare, Republic of Korea (HI18C2391); by the Basic Science Research Program through the National Research Foundation of Korea (NRF-2021R1A2C2005790, NRF-2020R1A2C1007789, NRF2019R1I1A1A01060496); by an NRF MRC grant funded by the Korean government (MSIP) (NRF-2018R1A5A2020732); and by a grant (2021IP0019 and 2019-098) from the Asan Institute for Life Sciences, Asan Medical Center, Seoul, Korea.

\section{Code Availability Not applicable.}

Author's Contributions All authors contributed to the study conception and design. Material preparation, data collection and analysis were performed by Hwan Yeul Yu, Jung Hyun Shin and HongDuck Yun. The first draft of manuscript was written by Jung Hyun Shin, Hwan Yeul Yu and Dong-Myung Shin and all authors commented on previous versions of the manuscript. All authors read and approved the final manuscript.

Data Availability The transcriptome data described in this study have been deposited in the Gene Expression Omnibus (GEO) of the NCBI and are accessible under GEO series accession number GSE122060 [19].

\section{Declarations}

Ethics Approval Animal experiments were approved by the Institutional Animal Care and Use Committee of the University of Ulsan College of Medicine (IACUC-2019-12-004) and performed in accordance with the guidelines and regulations.

\section{Consent to Participate Not applicable.}

\section{Consent for Publication Not applicable.}

Conflict of Interest None.

Open Access This article is licensed under a Creative Commons Attribution 4.0 International License, which permits use, sharing, adaptation, distribution and reproduction in any medium or format, as long as you give appropriate credit to the original author(s) and the source, provide a link to the Creative Commons licence, and indicate if changes were made. The images or other third party material in this article are included in the article's Creative Commons licence, unless indicated otherwise in a credit line to the material. If material is not included in the article's Creative Commons licence and your intended use is not permitted by statutory regulation or exceeds the permitted use, you will need to obtain permission directly from the copyright holder. To view a copy of this licence, visit http://creativecommons.org/licenses/by/4.0/.

\section{References}

1. Abrams, P., Cardozo, L., Fall, M., Griffiths, D., Rosier, P., Ulmsten, U., van Kerrebroeck, P., Victor, A., Wein, A., \& Standardisation Sub-committee of the International Continence Society. (2002). The standardisation of terminology of lower urinary tract function: Report from the standardisation subcommittee of the international continence society. Neurourology and Urodynamics, 21(2), 167-178.

2. Smith, P. P., Birder, L. A., Abrams, P., Wein, A. J., \& Chapple, C. R. (2016). Detrusor underactivity and the underactive bladder: Symptoms, function, cause-what do we mean? ICI-RS think tank 2014. Neurourology and Urodynamics, 35(2), 312-317.

3. Osman, N. I., Esperto, F., \& Chapple, C. R. (2018). Detrusor underactivity and the underactive bladder: A systematic review of preclinical and clinical studies. European Urology, 74(5), 633-643.

4. Shin, J. H., Ryu, C. M., Yu, H. Y., Shin, D. M., \& Choo, M. S. (2020). Current and future directions of stem cell therapy for bladder dysfunction. Stem Cell Reviews and Reports, 16(1), 82-93.

5. Kim, Y., Jin, H. J., Heo, J., Ju, H., Lee, H. Y., Kim, S., Lee, S., Lim, J., Jeong, S. Y., Kwon, J. H., Kim, M., Choi, S. J., Oh, W., Yang, Y. S., Hwang, H. H., Yu, H. Y., Ryu, C. M., Jeon, H. B., \& Shin, D. M. (2018). Small hypoxia-primed mesenchymal stem cells attenuate graft-versus-host disease. Leukemia, 32(12), 2672-2684.

6. E., H. K., Michelangelo, C., Kate, D., M., R. A., Filipa, V., KwanLeong, H., et al. (2018). Embryonic stem cell-derived mesenchymal stem cells (MSCs) have a superior neuroprotective capacity over fetal MSCs in the hypoxic-ischemic mouse brain. Stem Cells Translational Medicine, 7(5), 439-449.

7. Sheyn, D., Ben-David, S., Shapiro, G., De Mel, S., Bez, M., Ornelas, L., et al. (2016). Human induced pluripotent stem cells differentiate into functional mesenchymal stem cells and repair bone defects. Stem Cells Translational Medicine, 5(11), 14471460.

8. Zhang, Y., Liang, X., Liao, S., Wang, W., Wang, J., Li, X., Ding, Y., Liang, Y., Gao, F., Yang, M., Fu, Q., Xu, A., Chai, Y. H., He, J., Tse, H. F., \& Lian, Q. (2015). Potent paracrine effects of human induced pluripotent stem cell-derived mesenchymal stem cells attenuate doxorubicin-induced cardiomyopathy. Scientific Reports, 5, 11235 .

9. Kim, A., Yu, H. Y., Lim, J., Ryu, C. M., Kim, Y. H., Heo, J., Han, J. Y., Lee, S., Bae, Y. S., Kim, J. Y., Bae, D. J., Kim, S. Y., Noh, B. J., Hong, K. S., Han, J. Y., Lee, S. W., Song, M., Chung, H. M., Kim, J. K., Shin, D. M., \& Choo, M. S. (2017). Improved efficacy and in vivo cellular properties of human embryonic stem cell derivative in a preclinical model of bladder pain syndrome. Scientific Reports, $7(1), 8872$.

10. Ryu, C. M., Yu, H. Y., Lee, H. Y., Shin, J. H., Lee, S., Ju, H., Paulson, B., Lee, S., Kim, S., Lim, J., Heo, J., Hong, K. S., Chung, H. M., Kim, J. K., Shin, D. M., \& Choo, M. S. (2018). Longitudinal intravital imaging of transplanted mesenchymal stem cells elucidates their functional integration and therapeutic potency in an animal model of interstitial cystitis/bladder pain syndrome. Theranostics, 8(20), 5610-5624.

11. Lee, S. W., Ryu, C. M., Shin, J. H., Choi, D., Kim, A., Yu, H. Y., Han, J. Y., Lee, H. Y., Lim, J., Kim, Y. H., Heo, J., Lee, S., Ju, H., Kim, S., Hong, K. S., Han, J. Y., Song, M., Chung, H. M., Kim, J. K., Shin, D. M., \& Choo, M. S. (2018). The therapeutic effect of human embryonic stem cell-derived multipotent mesenchymal stem cells on chemical-induced cystitis in rats. International Neurourology Journal, 22(Suppl 1), S34-S45.

12. Shin, J. H., Ryu, C.-M., Ju, H., Yu, H. Y., Song, S., Hong, K.-S., Chung, H. M., Park, J., Shin, D. M., \& Choo, M. S. (2020). Therapeutic efficacy of human embryonic stem cell-derived 
multipotent stem/stromal cells in diabetic detrusor underactivity: A preclinical study. Journal of Clinical Medicine, 9(9).

13. Ryu, C. M., Shin, J. H., Yu, H. Y., Ju, H., Kim, S., Lim, J., Heo, J., Lee, S., Shin, D. M., \& Choo, M. S. (2019). N-acetylcysteine prevents bladder tissue fibrosis in a lipopolysaccharide-induced cystitis rat model. Scientific Reports, 9(1), 8134.

14. Song, M., Lim, J., Yu, H. Y., Park, J., Chun, J. Y., Jeong, J., Heo, J., Kang, H., Kim, Y. H., Cho, Y. M., Kim, S. W., Oh, W., Choi, S. J., Jang, S. W., Park, S., Shin, D. M., \& Choo, M. S. (2015). Mesenchymal stem cell therapy alleviates interstitial cystitis by activating Wnt signaling pathway. Stem Cells and Development, 24(14), 1648-1657.

15. Kim, A., Yu, H. Y., Heo, J., Song, M., Shin, J. H., Lim, J., Yoon, S. J., Kim, Y. H., Lee, S., Kim, S. W., Oh, W., Choi, S. J., Shin, D. M., $\&$ Choo, M. S. (2016). Mesenchymal stem cells protect against the tissue fibrosis of ketamine-induced cystitis in rat bladder. Scientific Reports, 6, 30881.

16. Shin, J. H., Ryu, C.-M., Ju, H., Yu, H. Y., Song, S., Shin, D.-M., \& Choo, M. S. (2019). Synergistic effects of N-acetylcysteine and mesenchymal stem cell in a lipopolysaccharide-induced interstitial cystitis rat model. Cells, 9(1), 86 .

17. Song, M., Heo, J., Chun, J.-Y., Bae, H. S., Kang, J. W., Kang, H., Cho, Y. M., Kim, S. W., Shin, D. M., \& Choo, M. S. (2013). The paracrine effects of mesenchymal stem cells stimulate the regeneration capacity of endogenous stem cells in the repair of a bladderoutlet-obstruction-induced overactive bladder. Stem Cells and Development, 23(6), 654-663.

18. Lim, J., Heo, J., Ju, H., Shin, J.-W., Kim, Y., Lee, S., et al. (2020). Glutathione dynamics determine the therapeutic efficacy of mesenchymal stem cells for graft-versus-host disease via CREB1-NRF2 pathway. Science Advances, 6(16), eaba1334.

19. Kim, M., Yu, H. Y., Ju, H., Shin, J. H., Kim, A., Lee, J., Ryu, C. M., Yun, H. D., Lee, S., Lim, J., Heo, J., Shin, D. M., \& Choo, M. S. (2019). Induction of detrusor underactivity by extensive vascular endothelial damages of iliac arteries in a rat model and its pathophysiology in the genetic levels. Scientific Reports, 9(1), 16328.

20. Heo, J., Noh, B. J., Lee, S., Lee, H. Y., Kim, Y., Lim, J., Ju, H., Yu, H. Y., Ryu, C. M., Lee, P. C., Jeong, H., Oh, Y., Kim, K., Kim, S. Y., Son, J., Hong, B., Kim, J. S., Cho, Y. M., \& Shin, D. M. (2020). Phosphorylation of TFCP2L1 by CDK1 is required for stem cell pluripotency and bladder carcinogenesis. EMBO Molecular Medicine, 12(1), e10880.

21. Heo, J., Lim, J., Lee, S., Jeong, J., Kang, H., Kim, Y., Kang, J. W., Yu, H. Y., Jeong, E. M., Kim, K., Kucia, M., Waigel, S. J., Zacharias, W., Chen, Y., Kim, I. G., Ratajczak, M. Z., \& Shin, D. M. (2017). Sirt1 regulates DNA methylation and differentiation potential of embryonic stem cells by antagonizing Dnmt31. Cell Reports, 18(8), 1930-1945.

22. Kang, H., Kim, K.-H., Lim, J., Kim, Y.-S., Heo, J., Choi, J., Jeong, J., Kim, Y. H., Kim, S. W., Oh, Y. M., Choo, M. S., Son, J., Kim, S. J., Yoo, H. J., Oh, W., Choi, S. J., Lee, S. W., \& Shin, D. M. (2015). The therapeutic effects of human mesenchymal stem cells primed with Sphingosine-1 phosphate on pulmonary artery hypertension. Stem Cells and Development, 24(14), 1658-1671.

23. Bastakoty, D., \& Young, P. P. (2016). Wnt/beta-catenin pathway in tissue injury: Roles in pathology and therapeutic opportunities for regeneration. The FASEB Journal, 30(10), 3271-3284.

24. Shin, K., Lee, J., Guo, N., Kim, J., Lim, A., Qu, L., Mysorekar, I. U., \& Beachy, P. A. (2011). Hedgehog/Wnt feedback supports regenerative proliferation of epithelial stem cells in bladder. Nature, 472(7341), 110-114.

25. Osman, N. I., Chapple, C. R., Abrams, P., Dmochowski, R., Haab, F., Nitti, V., Koelbl, H., van Kerrebroeck, P., \& Wein, A. J. (2014). Detrusor underactivity and the underactive bladder: A new clinical entity? A review of current terminology, definitions, epidemiology, aetiology, and diagnosis. European Urology, 65(2), 389-398.
26. Osman, N. I., \& Chapple, C. R. (2014). Contemporary concepts in the aetiopathogenesis of detrusor underactivity. Nature Reviews. Urology, 11(11), 639-648.

27. Bayrak, Ö., \& Dmochowski, R. R. (2019). Underactive bladder: A review of the current treatment concepts. Turkish Journal of Urology, 45(6), 401-409.

28. Aizawa, N., \& Igawa, Y. (2017). Pathophysiology of the underactive bladder. Investig Clin Urol, 58(Suppl 2), S82-S89.

29. Gilleran, J., Diokno, A. C., Ward, E., Sirls, L., Hasenau, D., Giordano, J., Shea, E., Bartolone, S. N., Lamb, L. E., \& Chancellor, M. B. (2021). Improved global response outcome after intradetrusor injection of adult muscle-derived cells for the treatment of underactive bladder. International Urology and Nephrology, 53, 1331-1338.

30. Zaim, M., Karaman, S., Cetin, G., \& Isik, S. (2012). Donor age and long-term culture affect differentiation and proliferation of human bone marrow mesenchymal stem cells. Annals of Hematology, 91(8), 1175-1186.

31. Kretlow, J. D., Jin, Y.-Q., Liu, W., Zhang, W. J., Hong, T.-H., Zhou, G., et al. (2008). Donor age and cell passage affects differentiation potential of murine bone marrow-derived stem cells. $B M C$ Cell Biology, 9(1), 60.

32. Wagner, W., Bork, S., Horn, P., Krunic, D., Walenda, T., Diehlmann, A., Benes, V., Blake, J., Huber, F. X., Eckstein, V., Boukamp, P., \& Ho, A. D. (2009). Aging and replicative senescence have related effects on human stem and progenitor cells. PLoS One, 4(6), e5846.

33. Lee, S., Lim, J., Lee, J. H., Ju, H., Heo, J., Kim, Y., Kim, S., Yu, H. Y., Ryu, C. M., Lee, S. Y., Han, J. M., Oh, Y. M., Lee, H., Jang, H., Yoon, T. J., Ahn, H. S., Kim, K., Kim, H. R., Roe, J. S., Chung, H. M., Son, J., Kim, J. S., \& Shin, D. M. (2020). Ascorbic acid 2glucoside stably promotes the primitiveness of embryonic and mesenchymal stem cells through ten-eleven translocation- and cAMPresponsive element-binding Protein-1-dependent mechanisms. Antioxidants \& Redox Signaling, 32(1), 35-59.

34. Moll, G., Rasmusson-Duprez, I., von Bahr, L., Connolly-Andersen, A.-M., Elgue, G., Funke, L., Hamad, O. A., Lönnies, H., Magnusson, P. U., Sanchez, J., Teramura, Y., Nilsson-Ekdahl, K., Ringdén, O., Korsgren, O., Nilsson, B., \& le Blanc, K. (2012). Are therapeutic human mesenchymal stromal cells compatible with human blood? Stem Cells, 30(7), 1565-1574.

35. Jeong, E. M., Yoon, J. H., Lim, J., Shin, J. W., Cho, A. Y., Heo, J., Lee, K. B., Lee, J. H., Lee, W. J., Kim, H. J., Son, Y. H., Lee, S. J., Cho, S. Y., Shin, D. M., Choi, K., \& Kim, I. G. (2018). Real-time monitoring of glutathione in living cells reveals that high glutathione levels are required to maintain stem cell function. Stem Cell Reports, 10(2), 600-614.

36. Chen, Q., Zhang, H., Liu, Y., Adams, S., Eilken, H., Stehling, M., Corada, M., Dejana, E., Zhou, B., \& Adams, R. H. (2016). Endothelial cells are progenitors of cardiac pericytes and vascular smooth muscle cells. Nature Communications, 7, 12422.

37. Gaskill, C. F., Carrier, E. J., Kropski, J. A., Bloodworth, N. C., Menon, S., Foronjy, R. F., Taketo, M. M., Hong, C. C., Austin, E. D., West, J. D., Means, A. L., Loyd, J. E., Merryman, W. D., Hemnes, A. R., de Langhe, S., Blackwell, T. S., Klemm, D. J., \& Majka, S. M. (2017). Disruption of lineage specification in adult pulmonary mesenchymal progenitor cells promotes microvascular dysfunction. The Journal of Clinical Investigation, 127(6), 2262-2276.

38. Lee, S., Elaskandrany, M., Lau, L. F., Lazzaro, D., Grant, M. B., \& Chaqour, B. (2017). Interplay between CCN1 and Wnt5a in endothelial cells and pericytes determines the angiogenic outcome in a model of ischemic retinopathy. Scientific Reports, 7(1), 1405.

39. Mazzoni, J., Smith, J. R., Shahriar, S., Cutforth, T., Ceja, B., \& Agalliu, D. (2017). The Wnt inhibitor Apcdd1 coordinates vascular 
remodeling and barrier maturation of retinal blood vessels. Neuron, 96(5), 1055-1069 e1056.

40. Nomiya, M., Yamaguchi, O., Akaihata, H., Hata, J., Sawada, N., Kojima, Y., \& Andersson, K. E. (2014). Progressive vascular damage may lead to bladder underactivity in rats. The Journal of Urology, 191(5), 1462-1469.

41. Sagawa, K., Aikawa, K., Nomiya, M., Ogawa, S., Akaihata, H., Takahashi, N., et al. (2013). Impaired detrusor contractility in a rat model of chronic bladder ischemia. Urology, 81(6), 1379 e1379-1314.

42. Lebovic, D. I., Bentzien, F., Chao, V. A., Garrett, E. N., Meng, Y. G., \& Taylor, R. N. (2000). Induction of an angiogenic phenotype in endometriotic stromal cell cultures by interleukin-1beta. Molecular Human Reproduction, 6(3), 269-275.

43. Zhou, W., Guo, S., \& Gonzalez-Perez, R. R. (2011). Leptin proangiogenic signature in breast cancer is linked to IL-1 signalling. British Journal of Cancer, 104(1), 128-137.

44. Kolb, R., Phan, L., Borcherding, N., Liu, Y., Yuan, F., Janowski, A. M., Xie, Q., Markan, K. R., Li, W., Potthoff, M. J., Fuentes-Mattei, E., Ellies, L. G., Knudson, C. M., Lee, M. H., Yeung, S. C. J., Cassel, S. L., Sutterwala, F. S., \& Zhang, W. (2016). Obesityassociated NLRC4 inflammasome activation drives breast cancer progression. Nature Communications, 7, 13007.
45. Cho, S. J., Kim, S. Y., Jeong, H. C., Cheong, H., Kim, D., Park, S. J., Choi, J. J., Kim, H., Chung, H. M., Moon, S. H., \& Cha, H. J. (2015). Repair of ischemic injury by pluripotent stem cell based cell therapy without Teratoma through selective photosensitivity. Stem Cell Reports, 5(6), 1067-1080.

46. Schwartz, S. D., Regillo, C. D., Lam, B. L., Eliott, D., Rosenfeld, P. J., Gregori, N. Z., Hubschman, J. P., Davis, J. L., Heilwell, G., Spirn, M., Maguire, J., Gay, R., Bateman, J., Ostrick, R. M., Morris, D., Vincent, M., Anglade, E., del Priore, L. V., \& Lanza, R. (2015). Human embryonic stem cell-derived retinal pigment epithelium in patients with age-related macular degeneration and Stargardt's macular dystrophy: Follow-up of two open-label phase 1/2 studies. Lancet, 385(9967), 509-516.

47. Schwartz, S. D., Hubschman, J. P., Heilwell, G., Franco-Cardenas, V., Pan, C. K., Ostrick, R. M., Mickunas, E., Gay, R., Klimanskaya, I., \& Lanza, R. (2012). Embryonic stem cell trials for macular degeneration: A preliminary report. Lancet, 379(9817), 713-720.

Publisher's Note Springer Nature remains neutral with regard to jurisdictional claims in published maps and institutional affiliations. 